\title{
Negative Posttraumatic Cognitions Color the Pathway from Event Centrality to Posttraumatic Stress Disorder Symptoms
}

\author{
Helen Johanßen ${ }^{1}(1) \cdot$ Nikola Schoofs $^{1} \cdot$ Reinhold Kliegl $^{2} \cdot$ Felix Bermpohl $^{1} \cdot$ Dominik Ülsmann $^{3}$. \\ Olaf Schulte-Herbrüggen ${ }^{3} \cdot$ Kathlen Priebe $^{1}$
}

Accepted: 2 September 2021 / Published online: 17 September 2021

(c) The Author(s) 2021

\begin{abstract}
Background The centrality of an event indicates the extent to which it becomes a core part of identity and life story. Event centrality (EC) has been shown to have a strong relationship with PTSD symptoms, which seems to be indirectly influenced by negative posttraumatic cognitions (PTC). However, research on this potential mediation and its causal links particularly with clinical samples is limited and essential to derive treatment implications.

Methods Pre- and posttreatment data of 103 day-unit patients with PTSD was examined using mediation analyses and structural equation modeling.

Results Negative PTC mediated the relationship between EC and PTSD symptoms, partially pre- and completely posttreatment. Within extended longitudinal analyses causal directions of the mediation pathways were not adequately interpretable due to unexpected suppression effects.

Conclusions The results suggest that EC may only have an indirect effect on PTSD symptoms through negative PTC. Thus, decreasing negative PTC which are connected to centralized events might be a key element for PTSD treatment. Thereby, transforming the cognitions' valence to more positive and constructive forms could be crucial rather than mere decentralization. Although suppression effects limited causal inferences, they do not contradict the mediation and further indicate potential interactional terms and a transformation of EC.
\end{abstract}

Keywords Event centrality $\cdot$ PTSD $\cdot$ Posttraumatic cognitions $\cdot$ Valence

Even though exposure to a traumatic event is very likely across countries with over $70.3 \%$, the conditional risk for posttraumatic stress disorder (PTSD) in the aftermath of trauma is only $4.0 \%$ cross-nationally (Kessler et al., 2017; Liu et al., 2017). This low probability has brought etiological factors for PTSD into the focus of research interest. A strong link has been established to posttraumatic cognitions (PTC) that are assumed to play a crucial role in etiology models of PTSD: Particularly views of individuals with strong beliefs about a safe world and high competences can

Helen Johanßen

helen.johanssen@charite.de

1 Department of Psychiatry and Psychotherapy, Charité Universitätsmedizin Berlin, 10117 Berlin, Germany

2 University of Potsdam, 14469 Potsdam, Germany

3 Friedrich Von Bodelschwingh-Klinik, Clinic for Psychiatry, Psychotherapy and Psychosomatic Medicine, 10717 Berlin, Germany be shattered through traumatic events since assimilation or over-accommodation of world- and self-schemes may lead to dysfunctional thoughts and consequently yield high vulnerability for PTSD (Foa \& Rothbaum, 1998). Similarly, Ehlers and Clark (2000) proposed in their cognitive model that in the aftermath of trauma a disturbance of autobiographical memory and negative appraisals of a trauma and/ or its consequences such as "My personality has changed for the worse" or "Nowhere is safe" (p. 322) lead to an ongoing perceived threat that can result in PTSD symptoms. Therefore, treatments such as cognitive therapy for PTSD (Ehlers et al., 2005) or cognitive processing therapy (Resick et al., 2017) aim to alter PTC. A recent review of treatment studies showed that the reduction of PTSD symptoms was often preceded by a decrease in negative PTC (Brown et al., 2019). In a commentary on 11 studies on appraisals and PTSD, McNally and Woud (2019) address the question if the relationship between trauma and PTSD is mediated by appraisals and evaluate methodological limitations for 
inferences. The authors outline that appraisals are found to be a correlate and predictor for PTSD but studies examining them as causal risk factor are rare. McNally and Woud (2019) reflect on innovations in this research field and point out that replicating previous results in clinical samples as well as causal research is important, particularly to derive therapeutical interventions.

Another facet of cognitions associated with PTSD symptomatology is the perceived centrality of a traumatic event, which arises if the event (1) is seen as a reference point for everyday inferences as well as (2) a turning point in life story and (3) becomes a core component of personal identity (Berntsen \& Rubin, 2006). Berntsen and Rubin $(2006,2007)$ argue that these three processes can emerge due to a high accessibility of traumatic memories through their emotional impact and distinctiveness. Thus, they assume an enhanced integration may be responsible for maladaptive cognitions and behavioral responses such as an overestimation of further traumatic events and avoidance of perceived threats. In a systematic review of 92 studies which mainly included student samples (Gehrt et al., 2018), a robust positive relationship between event centrality (EC) and PTSD symptoms was found. Beyond mainly cross-sectional evidence, only few studies have investigated longitudinal effects so far and suggest that EC precedes and predicts PTSD symptoms (Blix et al., 2016; Boals \& Ruggero, 2016; Boelen, 2012; Grau et al., 2020) while one recent study indicates the reversed temporal order (Glad et al., 2019). In line with the picture of multiple studies included in the review by Gehrt et al. (2018), previous research predominantly indicates that EC effects PTSD symptom severity.

Concurrently, the review by Gehrt et al. (2018) showed that EC correlated strongest with posttraumatic growth (PTG), which is described as a positive change after highly adverse life events and includes an increase of life appreciation, significant relationships, and perceived personal strength, as well as a shift of priorities and a development of an existential or spiritual life (Tedeschi \& Calhoun, 2004). Tedeschi and Calhoun (2004) bring up that PTG "may be accompanied by a reduction in distress" (p. 13) but also point out that PTG cannot be directly equated with wellbeing or less distress.

The ambiguity of EC correlates stimulated more research. Boals and Schuettler (2011) found that EC predicted both, PTG and PTSD symptoms. They concluded that the way of coping (problem-focused vs. avoidant) and the valence of interpretations (positive vs. negative perspective) determine the course of mental health outcomes (Schuettler \& Boals, 2011). Barton et al. (2013) came to the same conclusion, suggesting that negative PTC come along with PTSD symptoms and inhibit PTG. In line with these findings, Groleau et al. (2013) concluded that negative or positive outcomes after traumatic experiences might depend on the perceived valence of the centrality appraisal for the worse or better. Accordingly, an examination with a valence-modified version of an EC measure showed that central-negative appraisals were positively related to PTSD symptoms and negatively to PTG, whereas central-positive appraisals showed the inverse relationships (Teale Sapach et al., 2018). Broadbridge (2018) used a similar approach adding valencemodified items to the measurement and also found that centralizing events negatively predicted PTSD symptomatology much stronger than centralizing them positively.

The findings suggest that EC may not be connected directly to PTSD symptoms but indirectly through centrality-related, negative cognitions. Their potential impact has not been systematically investigated, but some studies have included examinations of mediational relationships: Using an undergraduate sample, Lancaster et al. (2011) found support for their hypothesis that EC mediated the relationship between PTC and PTSD symptoms and also found a good fit for a model with PTC as a mediator, concluding that both mediators are independent predictors. Two studies showed that violated core beliefs mediated the relationship between EC and PTSD symptoms in undergraduates (George et al., 2016) and internally displaced older adults (Chukwuorji et al., 2019). Vermeulen et al. (2019) were able to experimentally reduce students' centrality appraisals of distressing events and found no reduction of PTSD symptoms relatively to a placebo control training but demonstrated that PTC and rumination had a mediating effect. The authors concluded that "there might be no direct causal relation between appraisals of event centrality and symptoms of PTSD" (p. 223) and consider that EC does not encompass relevant appraisals based on PTSD models (e.g. by Ehlers \& Clark, 2000). These mediational studies support the idea that negative PTC have an indirect effect of EC on PTSD symptoms, but more research is needed to confirm this assumption.

In sum, numerous studies have shown a strong association of EC and PTSD symptoms, but questions still remain concerning the influence of negative PTC and causal directions of the relationships. These are core targets of this study. We aimed to study the associations of the three variables examining a clinical sample that had experienced a traumatic event and using longitudinal design. This approach substantially broadens research in a field where so far predominantly subclinical student samples who refer to their most stressful event have been investigated cross-sectionally.

First, we hypothesized that negative PTC mediate the relationship between EC and PTSD symptoms pre- and posttreatment. We also expected to find these mediations when the cross-lagged paths and stabilities between the two points of time are included. Second, we expected to find evidence for causal directions of the mediational pathways through longitudinal analyses, such that EC influences PTC and that both influence PTSD symptoms. Thus, comparing the three 
pairs of cross-lagged paths, we hypothesized that the regressions implying the mediational direction are larger than the regressions vice versa.

\section{Methods}

\section{Treatment}

Patients voluntarily reached out to the trauma-focused day-unit program at St. Hedwig-Hospital in Berlin of the Psychiatric University Hospital Charité. The department provides cognitive behavioral therapy to patients suffering from posttraumatic disorders. The program ran from 8:00 am to $3: 30 \mathrm{pm}$ on weekdays over an average of 7.94 treatment weeks $(S D=1.45)$ for the included patients. It comprised four sessions of individual therapy per week and daily group therapy, conducted by mental health professionals (psychologists, psychiatrists, and nurses) who obtained multiple supervisions weekly. The composition of the group varied throughout.

The treatment followed the cognitive processing therapy (CPT) manual by Resick et al. (2017). In addition, behavioral experiments were conducted and avoidance as well as safetyseeking behavior was monitored (Ehlers et al., 2005). Within the individual therapy, half of the sessions were conducted under guidance of one main therapist. Patients were encouraged to write an Impact Statement which encompasses their beliefs of reasons for the occurrence of the traumatic event and its influence on their beliefs about self, others, and the world-particularly in terms of safety, trust, control, power, esteem, and intimacy. Thereby, dysfunctional beliefs (stuck points) were identified and challenged through socratic dialogue with the help of the professional and worksheets. Besides, patients wrote a narrative of their most distressing trauma and repeatedly read it. If present and necessary, other traumatic events could be addressed after working on the index trauma. The standard program was individually adapted to the therapy goals of the patients. The specific topics were consolidated with the help of a co-therapist within the other half of individual sessions. Besides, group therapy addressed more general and commonly arising topics. It comprised psychoeducation, thought and behavioral analyses, challenging irrational thoughts, planning and evaluating behavioral experiments, and monitoring avoidance as well as safety-seeking behavior. The use of various worksheets was thereby practiced.

To be admitted, a preliminary session by a trained and experienced clinician (psychologist or psychiatrist) was conducted to evaluate the indication for the treatment with a semi-structured clinical interview based on the diagnostic criteria according to ICD-10 (WHO, 1992). This study included only patients who fulfilled clinically assessed
PTSD diagnosis (F43.1). To assure sufficient posttraumatic stress symptom severity, the criterion of the Posttraumatic Diagnostic Scale (PDS; Foa et al., 1997) had to be fulfilled additionally (at least one re-experiencing, three avoidance, and two hyperarousal symptoms had to be rated with 1 or higher and present over at least four weeks as well as the existence of at least one impairment).

\section{Patients}

From November 2014 to January 2020, 145 of 304 admitted patients completed the set of pre- (T1) and posttreatment (T2) assessments. Thereof, 109 patients fulfilled clinically assessed PTSD diagnosis, however six of them did not fulfill the PDS-criterion. Consequently, 103 patients ( 73 females; $70.9 \%$ ) were included in this study.

The average age of this sample was $40.46(S D=11.41)$, ranging from 20 to 63 years. Besides PTSD diagnosis, the majority of the patients $(n=85 ; 82.5 \%)$ were diagnosed with comorbid psychiatric disorders, mostly affective disorders $(n=70)$. Other comorbidities were substance use disorders $(n=15)$, anxiety disorders $(n=14)$, personality disorders $(n=8)$, dissociative (conversion) disorders $(n=4)$, somatoform disorders $(n=3)$, eating disorders $(n=2)$, and tic disorder $(n=1)$. The mean number of current disorders was $2.19(S D=0.86)$.

Most patients reported more than one traumatic event $(92.2 \%)$, ranging up to a maximum of $10(M=4.46$, $S D=2.07)$. Concerning the index trauma, the posttraumatic symptoms were in $48.5 \%$ of the patients related to sexual assault (childhood: $n=38$; adulthood: $n=9$; age unknown: $n=3$ ), in $20.4 \%$ to physical assault (childhood: $n=8$, adulthood: $n=7$; age unknown: $n=6$ ), in $19.4 \%$ to armed assault or combat experience or terror attack $(n=20)$, in $8.7 \%$ to accidents $(n=9)$, and in $2.9 \%$ to other incidents (witness of suicide or death; $n=3$ ).

\section{Measures}

Data acquisition was part of the routine clinical monitoring at the day-unit. The self-rating measures of this study were collected in German on a tablet at the week of admission and averagely 55.82 days $(S D=10.31)$ later at the week of discharge. The Posttraumatic Diagnostic Scale (PDS) was only assessed at admission because of its diagnostic value.

\section{Event Centrality}

The Centrality of Event Scale (CES; Berntsen \& Rubin, 2006; German version: Conen et al., 2021) measures to which extend an event becomes central to an individual, referring to its identity and life story. An event is seen as central if it becomes a (1) reference point, (2) turning point, 
and (3) central component of personal identity. The authors introduced a questionnaire with 20 items $(\alpha=0.94)$ and a shortened version $(\alpha=0.88)$ with seven items of the long version (no. 3, 6, 10, 12, 16, 17, and 18). Both scales are rated on a 5-point Likert-scale, ranging from 1 (totally disagree) to 5 (totally agree). In this study, the short version was used, which demonstrated acceptable internal consistency at admission $(\alpha=0.78)$ and good internal consistency at discharge $(\alpha=0.88)$.

\section{Posttraumatic Cognitions}

The Posttraumatic Cognitions Inventory (PTCI; Foa et al., 1999; German version: Ehlers \& Boos, 2000) was used to assess trauma-related thoughts and beliefs. It consists of the three factors: Negative cognitions about self (21 items), negative cognitions about the world (7 items), and self-blame (5 items). Rated on a 7-point Likert-scale from 1 (totally disagree) to 7 (totally agree), high scale scores indicate stronger endorsement of negative cognitions. All factors as well as the total score showed excellent internal consistency $(\alpha=0.88-0.97$; Foa et al., 1999). Internal consistency in this sample proved excellent reliability at admission $(\alpha=0.94)$ and discharge $(\alpha=0.98)$.

\section{Posttraumatic Stress Symptoms}

The Posttraumatic Diagnostic Scale (PDS; Foa et al., 1997; German version: Ehlers et al., 1996) measures the presence of posttraumatic stress disorder (PTSD) and the severity of its symptoms within the past month. The four parts of the questionnaire are allocated to the criteria of PTSD according to the Diagnostic and Statistical Manual of Mental Disorders (DSM-IV; APA, 1994): Part 1 and 2 assess trauma history and characteristics of the traumatic events (types, index event, time of occurrence, circumstances and reactions). Part 3 measures the PTSD symptom clusters (intrusions, avoidance, hyperarousal) and their frequency with 17 items, ranging from 0 (not at all or only one time) to 3 (five or more times a week/almost always) on a 4-point Likertscale. Two further questions assess the duration and onset of symptoms. In Part 4, impairments as a result of the PTSD symptoms are retrieved. The total symptom severity (Part 3) demonstrated with $\alpha=0.92$ an excellent internal consistency (Foa et al., 1997) while it was acceptable $(\alpha=0.76)$ in the present sample.

Comparable to Part 3 of the PDS, the Davidson Trauma Scale (DTS; Davidson et al., 1997) measures the presence of PTSD symptoms according to DSM-IV (APA, 1994). Consisting of 17 items, both the frequency and severity of the PTSD symptom clusters were rated on a 5-point Likert-scale, ranging from not at all (0) to every day (4) and not at all distressing (0) to extremely distressing (4). The DTS showed good internal consistency $(\alpha=0.99)$ and is sensitive to treatment effects (Davidson et al., 1997). In the present study, internal consistencies were excellent at admission $(\alpha=0.92)$ and discharge $(\alpha=0.96)$.

\section{Statistical Analyses}

Analyses were conducted using R version 3.6.3 (R Core Team, 2020) and are presented in the Appendix A. For descriptive statistics, one sample Kolmogorov-Smirnov-Tests were conducted to test normal distributions, paired $t$-tests to investigate the mean changes over time, and Pearson correlations to examine the relationships between the variables. Cohen`s $d$ was calculated as effect size (Cohen, 1988).

Mediations and the structural equation model (SEM) were estimated with the lavaan package (Rosseel, 2012). To test the mediation model regarding the first hypothesis, the presence of indirect effect of negative PTC on the relationship between EC and PTSD symptoms was estimated separately as well as simultaneously for both points of time within one SEM using the Maximum-Likelihood method.

To examine the second hypothesis regarding the causal direction of the mediational pathways, such that EC influences PTC and that both influence PTSD symptoms, the three pairs of cross-lagged paths between the variables of each point of time were analyzed within the SEM comparing the sizes of their regression weights. We tested whether the regressions of (1) PTSD symptoms at discharge on EC at admission, (2) of PTC at discharge on EC at admission, and (3) PTSD symptoms at discharge on PTC at admission are larger than the respective regressions vice versa. Two crosslagged paths were considered to be different if a model with equality constraints on the paths fit the data significantly worse than the original unconstrained model. Models were compared using likelihood ratio tests with significant $\chi^{2}$ values indicating differences between the two cross-lagged paths.

To analyze power of the regression coefficients, the mixedDesign() function (Schad et al., 2020) was used: First, data for 1010 simulations was generated with the means, standard deviations and correlations from the specified model. Then, coefficients were extracted, rows with missing values excluded and 1000 rows randomly extracted. Last, the significant results were counted to estimate the power of the indirect effects and cross-lagged paths.

\section{Results}

\section{Descriptive Statistics}

The assumption of normally distributed scores was violated only for CES at discharge $(p<0.05)$. An analysis 
of changes from admission to discharge showed significant reductions $(p<0.001)$ for CES, $t(102)=5.71$, PTCI, $t(102)=9.47$, and DTS, $t(102)=8.57$. Effect sizes of the mean differences were medium for the CES $(d=0.56)$ and large for the PTCI $(d=0.93)$ and DTS $(d=0.84)$. The descriptive statistics and correlations are summarized in Table 1. All correlations were positive and significant at $p<0.001$. Cross-sectionally, correlations were of medium $(r=0.36-0.44)$ and large $(r=0.54-0.79)$ magnitudes at admission and discharge, respectively (Cohen, 1988).

\section{Cross-sectional Mediation}

According to the first hypothesis, negative PTC mediated the effect of EC on PTSD symptoms at admission (indirect effect: $z=2.55, p<0.05$ ) and discharge (indirect effect: $z=6.26, p<0.001)$. A partial mediation occurred at admission (direct effect: $z=3.64, p<0.001$ ), whereas it was complete at discharge (direct effect: $z=0.95$, $p=0.343$ ). Figure 1 presents the separate mediation models pre- and posttreatment with standardized regression coefficients.

\section{Structural Equation Modeling}

Figure 2 displays the SEM integrating the two mediation models of Fig. 1 along with standardized regression coefficients. Within this model, indirect effects of EC on PTSD symptoms via negative PTC were tested simultaneously and occurred at admission $(z=2.55, p<0.05)$ and discharge $(z=5.92, p<0.001)$ in line with the first hypothesis. Again, partial mediation occurred at admission (direct effect: $z=3.64, p<0.001$ ), whereas a complete mediation occurred at discharge (direct effect: $z=-0.63, p=0.529$ ). The analysis of power revealed high values for the indirect effects at admission $(84.3 \%)$ and discharge (100.0\%).

Stabilities $\left(s_{11}, s_{22}, s_{33}\right)$ of the questionnaires between the two points of time were throughout positive and significant. The power of the cross-lagged paths ( $c l p)$ analogous to the hypothesized direction of mediation paths was high $\left(c l p_{12}=89.3 \%, c l p_{23}=97.3 \%\right)$ and small $\left(c l p_{13}=33.6 \%\right)$, while the power of the cross-lagged paths inversed to the hypothesized mediation pathways was medium $\left(c l p_{31}=55.4 \%\right)$ and small $\left(c l p_{21}=37.4 \%, c l p_{32}=40.7 \%\right)$. Thus, the probability detecting a real effect of the path coefficients as statistically significant by the analysis was adequate for half of the clps with power above 50\%. Besides this descriptive information, interpreting the comparisons of clp coefficients was still appropriate since our second hypotheses focused on the coefficient sizes. As we hypothesized that EC influences PTC and that both influence PTSD symptoms, we expected larger coefficient sizes of these clp directions than reversed. Thus, analyses were performed comparing the standardized regression weights of the three paired crosslagged paths. The results showed patterns contrary to the expectations: First, the standardized regression of PTSD symptoms (T2) on EC (T1) was smaller $\left(c l p_{13}=0.09\right)$ than vice versa $\left(c l p_{31}=0.20\right), \chi_{\text {diff }}^{2}=1.60$, but the difference did not reach statistical significance, $p=0.206$. Second, the regression of PTC (T2) on EC (T1) was significantly smaller $\left(c l p_{12}=-0.23\right)$ compared to the reverse regression $\left(c l p_{21}=0.16\right), \chi_{\text {diff }}^{2}=9.74, p<0.01$. Lastly, the estimated coefficient of the regression of PTSD symptoms (T2) on PTC (T1) was also significantly smaller $\left(c l p_{23}=-0.27\right)$ than vice versa $\left(c l p_{32}=0.12\right), \chi_{\text {diff }}^{2}=8.20, p<0.01$. Overall, the expected larger regression coefficients of paths implying the mediational directions compared to paths of the reversed direction did not appear within the longitudinal analyses of this model.

Regarding the comparison of the cross-lagged paths, two negative regressions occurred $\left(c l p_{12}\right.$ and $\left.c l p_{23}\right)$, indicating unexpected suppression effects: EC at admission correlated higher with PTC at admission $(r=0.36)$ than at discharge $(r=0.22)$, implying that EC suppressed variance in PTC at admission which may have led to a high partial correlation of PTC at admission and discharge $(r=0.61)$. Similarly, PTC at admission correlated higher with PTSD symptoms at admission $(r=0.42)$ than at discharge $(r=0.38)$, indicating suppressed variance which may have led to a high partial correlation of PTSD symptoms at admission and discharge $(r=0.53)$. Thus, the subtractive contribution of EC as well
Table 1 Descriptive statistics and pearson correlations

\begin{tabular}{|c|c|c|c|c|c|c|c|c|}
\hline & & \multirow[b]{2}{*}{$M(S D)$} & \multicolumn{3}{|l|}{$\mathrm{T} 1$} & \multicolumn{3}{|l|}{$\mathrm{T} 2$} \\
\hline & & & CES & PTCI & DTS & CES & PTCI & DTS \\
\hline \multirow[t]{3}{*}{$\mathrm{T} 1$} & CES & $28.94(4.62)$ & - & & & & & \\
\hline & PTCI & $149.18(34.32)$ & $0.36 * * *$ & - & & & & \\
\hline & DTS & $83.31(22.13)$ & $0.44 * * *$ & $0.42 * * *$ & - & & & \\
\hline \multirow[t]{3}{*}{$\mathrm{T} 2$} & CES & $25.60(6.34)$ & $0.45 * * *$ & $0.35 * * *$ & $0.40 * * *$ & - & & \\
\hline & PTCI & $114.89(45.66)$ & $0.22 * * *$ & $0.61 * * *$ & $0.42 * * *$ & $0.63 * * *$ & - & \\
\hline & DTS & $61.59(29.27)$ & $0.28 * * *$ & $0.38 * * *$ & $0.53 * * *$ & $0.54 * * *$ & $0.79 * * *$ & - \\
\hline
\end{tabular}

CESCentrality of Event Scale, PTCIPosttraumatic Cognitions Inventory, DTS Davidson Trauma Scale, $T 1$ admission, $T 2$ discharge. $* * * p<0.001$ 
Fig. 1 Mediation analyses at admission (left) and at discharge (right). CESCentrality of Event Scale, PTCIPosttraumatic Cognitions Inventory, DTS Davidson Trauma Scale. ${ }^{* *} p<0.01$. $* * * p<0.001$
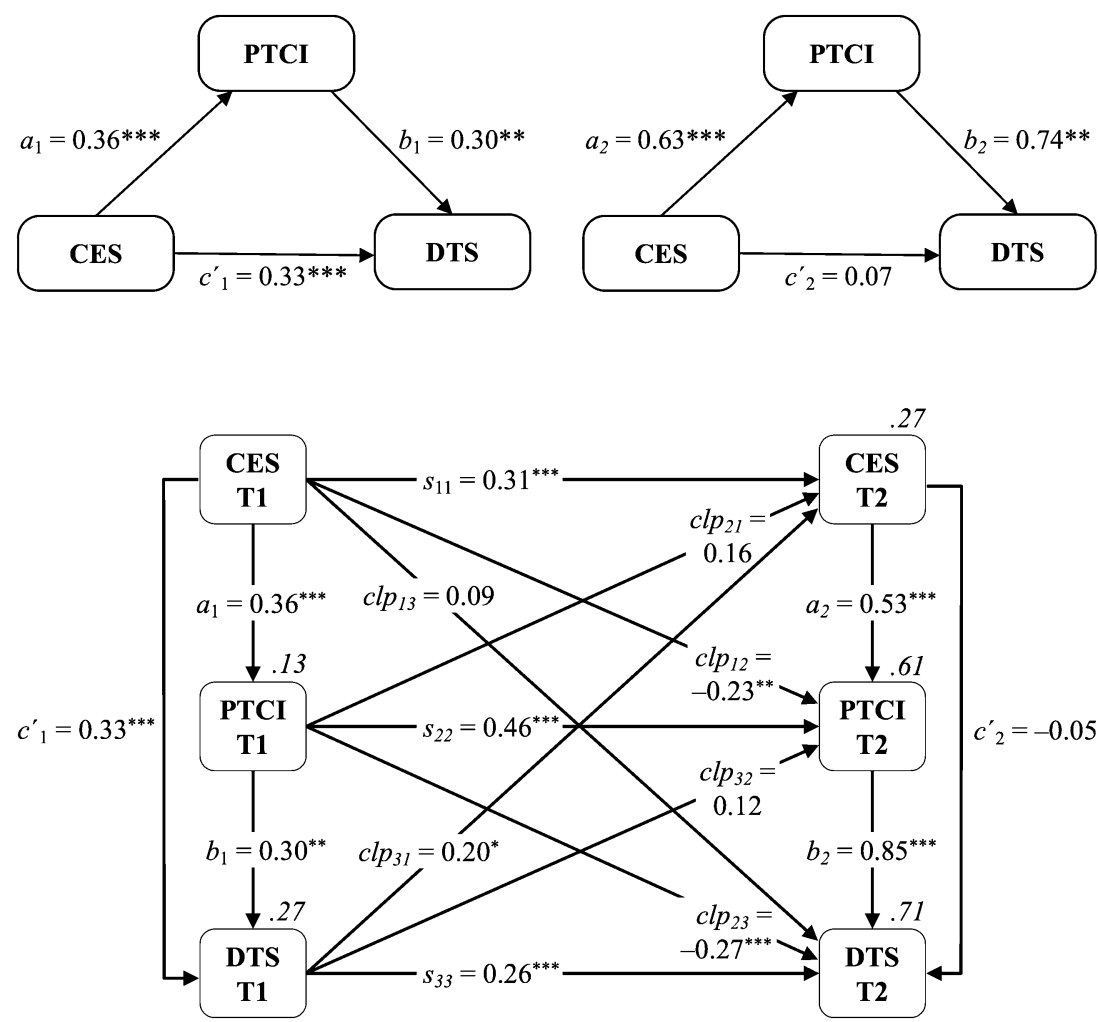

Fig. 2 Maximal Model displaying the Standardized Regression Coefficients and $\mathrm{R}^{2}$ in italics. CESCentrality of Event Scale, PTCIPosttraumatic Cognitions Inventory, DTS Davidson Trauma Scale, $T 1$ admission, $T 2$ discharge. ${ }^{*} p<0.05$ $* * p<0.01$. *** $p<0.001$ as PTC may be expressed in the negative regression coefficients. Due to the assumed suppression effects, the usual interpretations comparing two cross-lagged paths is not appropriate. For example, $c l p_{12}=-0.23$ cannot imply that higher EC values at admission were associated with lower PTC values at discharge, but rather reflects an increase of the positive stability coefficient of PTC.

\section{Discussion}

This study cross-sectionally and longitudinally investigated the relationship between EC and PTSD symptoms and the possible indirect effect of negative PTC on their relationship. To our knowledge, this is the first longitudinal study on this topic including a clinical sample. So far, previous research indicated that EC and negative PTC precede PTSD symptoms (Blix et al., 2016; Boelen, 2012; Brown et al., 2019; Grau et al., 2020) and centralizing events negatively resulted in higher symptomatology (Broadbridge, 2018; Teale Sapach et al., 2018). In addition, related mediational effects were found within students samples cross-sectionally (George et al., 2016; Lancaster et al., 2011) and longitudinally (Vermeulen et al., 2019) as well as in a sample of internally displaced older adults cross-sectionally (Chukwuorji et al., 2019).

Therefore, we first hypothesized that negative PTC mediate the relationship between EC and PTSD symptoms in a clinical sample pre- and posttreatment. Cross-sectionally, mediation was partial at admission and even complete at discharge, strengthening the evidence for the indirect effect. Within a longitudinal SEM including the cross-lagged paths and stabilities between the two points of time, we found the same mediational pattern. These results extend the findings of the separate tested mediations in support of the hypothesis since the stabilities from admission to discharge-even though they were comparably small but still positive as expected-were included within the tested model.

Concerning the second hypothesis, we expected to find extending evidence for the causal directions of the mediational pathways, such that EC influences PTC and that both influence PTSD symptoms. To investigate each direction of the paths, we compared the paired cross-lagged paths within the longitudinal design and expected that regression coefficients of paths implying the mediational directions are larger compared to the respective paths of the reversed direction. Against our assumptions, none of the three comparisons showed the expected pattern: The regression weight of PTSD symptoms at discharge on EC at admission was smaller than vice versa but the comparison was not significant, indicating no difference between the regressions. The regression of PTC at discharge on EC at admission as well as the regression of PTSD symptoms at discharge on PTC at admission were significantly smaller than the respective regressions vice versa. However, two negative coefficients within the cross-lagged paths led to the assumption of 
unexpected suppression effects which generally implicate a restriction of comparative interpretations. Thus, designating the cross-lagged paths as larger or smaller as mentioned above was considered inappropriate. Since causal inferences were therefore limited, the results cannot speak for or against reversed directions of the mediational pathways. Thereby, they do not contradict our previous findings.

Overall, the first part of our results regarding the mediation pre- and posttreatment implicate that particularly reducing negative PTC which are connected to centralized events might be essential for PTSD interventions. At the same time, this would suggest that reducing EC within trauma-focused interventions may not be directly relevant for achieving a reduction of PTSD symptoms. This would stand in contrast to the original assumption of a direct influence (Berntsen $\&$ Rubin, 2006) and recent suggestions (Boals et al., 2020) pleading for interventions which aim to specifically reduce EC. We assume that this may only have an effect on PTSD symptoms if the intervention implicitly targets connected, negative PTC. As mentioned above, the valence of centrality-related cognitions is not included in the Centrality of Event Scale (Berntsen \& Rubin, 2006). For example, an item asks to which extend the event has colored the way of thinking and feeling about other experiences—but it is not defined how, and the "color" remains unclear. Assimilation or overaccommodation processes in the aftermath of trauma, developing appraisals such as "The world is dangerous and an unsafe place", "I am weak" or "I cannot trust anyone", may lead to an ongoing perceived threat and avoidance behavior (Ehlers \& Clark, 2000). Whereas outlasting thoughts such as "I might not be able to control everything, but I can still control a lot" or "I can overcome challenges in life, surviving has made me a stronger person" might lead to a less negative, anxious "color" and even enhance PTG (Tedeschi $\&$ Calhoun, 2004). Either way, comprising a negative or positive valence, it seems conceivable that the event can be seen as and remain central in terms of an identity component, reference and turning point based on the concept of Berntsen and Rubin (2006). Our descriptive results indicate the reduction of EC over time was comparably smaller in terms of a medium effect size in comparison to large effect sizes concerning the reduction of negative PTC and PTSD symptoms. Also, the low stability coefficient of EC from admission to discharge may indicate a shift in its meaning over time which could indicate a transition of the latent construct from a negative to more positive centralization. These aspects may underline that instead of decentralization, reducing the negative valence of connected cognitions might be more essential. As we derive this suggestion from our results, we could assume that less negative PTC imply more positive PTC which are related to less PTSD symptoms. However, we did not specifically include a positive facet of PTC and can only speculate on the influence of this concept. With regard to results of studies that suggest having events centralized positively leads to good mental health outcomes (e.g. Broadbridge, 2018; Groleau et al., 2013; Schuettler \& Boals, 2011; Teale Sapach et al., 2018), focusing on the reduction of negative and a shift to positive appraisals of centralized events could lead to better outcomes. This would underline the importance of decreasing negative PTC on the one hand and enhancing positive PTC on the other hand as targets of PTSD treatments. In a clinical context, this could implicate utilizing cognitive techniques to reduce dysfunctional negative appraisals which have occurred in the aftermath of a trauma. In addition, individuals could be encouraged to develop positive appraisals for example being a strong person who is able to cope with a traumatic event or even to find positive effects such as strengthened relationships or changed priorities. Further research on positive PTC and its meanings would therefore be necessary to claim such inferences. Also, an additional orientation on growth instead of only focusing on deficits may be a helpful tool, since PTG can be associated with less distress (Tedeschi \& Calhoun, 2004). However, debates about whether PTG is rather related to negative mental health outcomes should be considered investigating this topic (e.g. Engelhard et al., 2015; Frazier et al., 2009).

Regarding the longitudinal results of our second part, the unexpected suppression effects restricted causal inferences as described above but despite implicate other valuable inferences: They could indicate interactional terms which were not represented within our model. For instance, EC may implicitly include negative or positive "colored" cognitions. Conversely, negative PTC might depend on the presence of EC and even strengthen it. Studies using valence-modified versions of the CES (Broadbridge, 2018; Teale Sapach et al., 2018) showing that higher PTSD symptom severity was present in case of a "negative" centralization underscore such a potential interaction. Similarly, negative PTC and PTSD symptomatology are also closely connected. For example, thinking the world is dangerous or being a weak person can certainly increase hypervigilance or avoidance behavior which in turn can maintain dysfunctional thoughts. The latest revision of the DSM underscores this idea including negative thoughts and feelings in PTSD criteria (DSM-5; APA, 2013). As it is thereby seen as a symptom and an integral part of the disorder, our model assuming PTC to have a mediational effect on PTSD symptoms is not necessarily suitable. In this light, investigating further cognitive aspects or processes leading to the symptom clusters of PTSD within extended structural models might be more appropriate.

Another aspect considering the longitudinal analyses is that in line with previous research (for an overview see Gehrt et al., 2018), the ratio of correlation sizes was similar to ours 
at admission but not at discharge, since it changed from preto posttest. For instance, while the correlation between EC and PTSD symptoms was the highest at admission, it was the lowest correlation at discharge. Again, this could indicate a shift in the meaning of EC, possibly from a negative to positive centralization. In case of a transition of a latent construct such as EC, lagged analyses may not be appropriate from a post-hoc perspective.

One methodological aspect of the longitudinal analysis is that it could be argued that the suppressor constellation absorbed a lot of error variance and might also have helped the mediation pattern to become visible more clearly, resulting in the change from a partial mediation at admission to a complete mediation at discharge. Thus, extended post-hocanalyses comparing models excluding all or only the negative cross-lagged paths did not support this explanation since the mediation patterns were still present (see Appendix B).

We see three notable strengths of this work which jointly stand out considering previous studies. First, we investigated a clinical sample with PTSD suffering from substantial posttraumatic symptomatology. Also, the patients experienced traumatic events of a diverse range, extending generalizability. Second, even though parts of our analyses regarding the comparability of the cross-lagged paths were restricted due to suppression effects, the longitudinal analyses gave more insights regarding potential interactional terms and a possible transformation of the latent EC construct. Third, our methodological approach was overall valuable due to the conduction of power analyses, SEM, and taking account of possible suppression effects which are often neglected.

At the same time, the suppression effects represent a methodological limitation of this study. They can often restrict SEMs as in the present case and are also difficult to replicate (e.g. Ghiselli, 1972). Another methodological constraint is that even though indirect effects can be statistically confirmed, we cannot rule out alternative mediators and reversed pathways; thus, the direction of the causality is not definable (Fiedler et al., 2011). Furthermore, even if the cross-lagged paths would have been interpretable and had given indications for temporal orders, more than two points of time are generally necessary for inference about causal directions in cross-lagged panels. Lastly, self-reported measures generally can cause a bias.

In conclusion, our results indicate that reducing negative PTC of centralized events should be a core target for the treatment of PTSD. Thereby, reframing in terms of a transformation of the cognition's "color" from negative (maladaptive) toward positive (constructive) appraisals could play a more important role than mere decentralization. Thus, the use of valence modified questionnaires proposed by Broadbridge (2018) or Teale Sapach et al. (2018) might bring more distinct insights when assessing improvements during therapy: Besides a reduction in negative PTC a shift to more positive appraisals might be essential.

Future research is necessary to confirm the mediational relationship between EC and PTSD symptoms via negative PTC. Thereby, potential interaction terms should be addressed. Future studies could also test with latent constructs whether there is a change in the meaning of EC reflected in different weights of measured variables at preand posttest. Ideally, further examinations should generally include more than two assessments to conclude possible causal influences. The inclusion of other associations that may impact the relationship of the variables, specifically the positive facet of PTC and PTG, could be complemented in order to get additional insights.

Supplementary Information The online version contains supplementary material available at https://doi.org/10.1007/s10608-021-10266-w.

Funding Open Access funding enabled and organized by Projekt DEAL. This study was not funded by any grants.

Data Availability $\mathrm{R}$ analyses are available in the Appendix. The data are not publicly available due to containing information that could comprise the privacy of participants.

\section{Declarations}

Conflict of interest The authors declare that they have no conflicts of interest.

Ethical Approval Data collection and analysis was approved by the ethical commission of the Psychiatric University Hospital Charité

Informed Consent Informed consent was obtained from all participants of this study, including the publication of the data.

Animal Rights This article does not contain any studies with animals performed by any of the authors.

Open Access This article is licensed under a Creative Commons Attribution 4.0 International License, which permits use, sharing, adaptation, distribution and reproduction in any medium or format, as long as you give appropriate credit to the original author(s) and the source, provide a link to the Creative Commons licence, and indicate if changes were made. The images or other third party material in this article are included in the article's Creative Commons licence, unless indicated otherwise in a credit line to the material. If material is not included in the article's Creative Commons licence and your intended use is not permitted by statutory regulation or exceeds the permitted use, you will need to obtain permission directly from the copyright holder. To view a copy of this licence, visit http://creativecommons.org/licenses/by/4.0/.

\section{References}

American Psychiatric Association. (1994). Diagnostic and statistical manual of mental disorders (4th ed.). Author. 
American Psychiatric Association. (2013). Diagnostic and statistical manual of mental disorders (DSM-5®). Author.

Barton, S., Boals, A., \& Knowles, L. (2013). Thinking about trauma: The unique contributions of event centrality and posttraumatic cognitions in predicting PTSD and posttraumatic growth. Journal of Traumatic Stress, 26, 718-726. https://doi.org/10.1002/jts. 21863

Berntsen, D., \& Rubin, D. C. (2006). The Centrality of Event Scale: A measure of integrating a trauma into one'sidentity and its relation to post-traumatic stress disorder symptoms. Behaviour Research and Therapy, 44(2), 219-231. https://doi.org/10.1016/j.brat.2005. 01.009

Berntsen, D., \& Rubin, D. C. (2007). When a trauma becomes a key to identity: Enhanced integration of trauma memories predicts posttraumatic stress disorder symptoms. Applied Cognitive Psychology, 21, 417-431. https://doi.org/10.1002/acp.1290

Blix, I., Birkeland, M. S., Solberg, Ø., Hansen, M. B., \& Heir, T. (2016). The launching and ensnaring effects of construing a traumatic event as central to one's identity and life story. Applied Cognitive Psychology, 30(4), 526-531. https://doi.org/10.1002/ acp. 3224

Boals, A., Griffith, E., \& Southard-Dobbs, S. (2020). A call for intervention research to reduce event centrality in trauma-exposed individuals. Journal of Loss and Trauma. https://doi.org/10.1080/ 15325024.2020 .1734744

Boals, A., \& Ruggero, C. (2016). Event centrality prospectively predicts PTSD symptoms. Anxiety Stress and Coping, 29, 533-541. https://doi.org/10.1080/10615806.2015.1080822

Boals, A., \& Schuettler, D. (2011). A double-edged sword: Event centrality, PTSD, and posttraumatic growth. Applied Cognitive Psychology, 25, 817-822. https://doi.org/10.1002/acp.1753

Boelen, P. A. (2012). A prospective examination of the association between the centrality of a loss and post-loss psychopathology. Journal of Affective Disorders, 137, 117-124. https://doi.org/10. 1016/j.jad.2011.12.004

Broadbridge, C. L. (2018). Is the centralization of potentially traumatic events always negative? An expansion of the Centrality of Events Scale. Applied Cognitive Psychology, 32(3), 315-325. https://doi. org/10.1002/acp.3403

Brown, A. L., Belli, G. M., Asnaani, A., \& Foa, E. B. (2019). A review of the role of negative cognitions about oneself, others, and the world in the treatment of PTSD. Cognitive Therapy and Research, 43, 143-173. https://doi.org/10.1007/s10608-018-9938-1

Chukwuorji, J. B. C., Ifeagwazi, C. M., \& Eze, J. E. (2019). Event centrality influences posttraumatic stress disorder symptoms via core beliefs in internally displaced older adults. Aging \& Mental Health, 23(1), 113-121. https://doi.org/10.1080/13607863.2017. 1396580

Cohen, J. (1988). Statistical power analysis for the behavioral sciences. Erlbaum.

Conen, L., Johanßen, H., Ülsmann, D., Ertle, A., Schulte, S., Fydrich, T., \& Schulte-Herbrüggen, O. (2021). Validation of a German translation of the Centrality of Event Scale (CES-G). [Manuscript in preparation].

Davidson, J. R., Book, S. W., Colket, J. T., Tupler, L. A., Roth, S., David, D., \& Feldman, M. E. (1997). Assessment of a new self-rating scale for posttraumatic stress disorder. Psychological Medicine, 27(1), 153-160. https://doi.org/10.1017/S0033 291796004229

Ehlers, A., \& Boos, A. (2000). Fragebogen zu Gedanken nach traumatischen Erlebnissen (PTCI). In A. Ehlers (Ed.), Posttraumatische Belastungsstörungen. Göttingen: Hogrefe.

Ehlers, A., \& Clark, D. M. (2000). A cognitive model of posttraumatic stress disorder. Behaviour Research and Therapy, 38, 319-345. https://doi.org/10.1016/S0005-7967(99)00123-0
Ehlers, A., Clark, D. M., Hackmann, A., McManus, F., \& Fennell, M. (2005). Cognitive therapy for posttraumatic stress disorder: development and evaluation. Behaviour Research and Therapy, 43(4), 413-431. https://doi.org/10.1016/j.brat.2004.03.006

Ehlers, A., Steil, R., Winter, H., \& Foa, E. B. (1996). Deutsche Übersetzung der Posttraumatic Stress Symptom Scale (PDS). University, Warneford Hospital.

Engelhard, I. M., Lommen, M. J. J., \& Sijbrandij, M. (2015). Changing for better or worse? Posttraumatic growth eported by soldiers deployed to Iraq. Clinical Psychological Science, 3(5), 789-796. https://doi.org/10.1177/2167702614549800

Fiedler, K., Schott, M., \& Meiser, T. (2011). What mediation analysis can (not) do. Journal of Experimental Social Psychology, 47, 1231-1236. https://doi.org/10.1016/j.jesp.2011.05.007

Foa, E. B., Cashman, L., Jaycox, L., \& Perry, K. (1997). The validation of a self-report measure of posttraumatic stress disorder: The Posttraumatic Diagnostic Scale. Psychological Assessment, 9, 445-451. https://doi.org/10.1037/1040-3590.9.4.445

Foa, E. B., Ehlers, A., Clark, D. M., Tolin, D. F., \& Orsillo, S. M. (1999). The Posttraumatic Cognitions Inventory (PTCI): Development and validation. Psychological Assessment, 11(3), 303-314.

Foa, E. B., \& Rothbaum, B. O. (1998). Treating the trauma of rape: Cognitive behavioral therapy for PTSD. Guilford Press.

Frazier, P., Tennen, H., Gavian, M., Park, C., Tomich, P., \& Tashiro, T. (2009). Does self-reported posttraumatic growth reflect genuine positive change? Psychological Science, 20(7), 912-919. https:// doi.org/10.1111/j.1467-9280.2009.02381.x

Gehrt, T. B., Berntsen, D., Hoyle, R. H., \& Rubin, D. C. (2018). Psychological and clinical correlates of the Centrality of Event Scale: A systematic review. Clinical Psychology Review, 65, 57-80. https://doi.org/10.1016/j.cpr.2018.07.006

George, L. S., Park, C. L., \& Chaudoir, S. R. (2016). Examining the relationship between trauma centrality and posttraumatic stress disorder symptoms: A moderated mediation approach. Traumatology, 22, 85-93. https://doi.org/10.1037/trm0000063

Ghiselli, E. E. (1972). Comment on the use of moderator variables. Journal of Applied Psychology, 56(3), 270. https://doi.org/10. 1037/h0033091

Glad, K. A., Czajkowski, N. O., Dyb, G., \& Hafstad, G. S. (2019). Cross-lagged association between symptoms of posttraumatic stress disorder and perceived centrality of a terrorist attack. Clinical Psychological Science, 8(2), 295-305. https://doi.org/10.1177/ 2167702619873590

Grau, P. P., Larsen, S. E., Lancaster, S. L., Garnier-Villarreal, M., \& Wetterneck, C. T. (2020). Change in event centrality and posttraumatic stress disorder symptoms during intensive treatment. Journal of Traumatic Stress, 31(1), 116-123. https://doi.org/10. $1002 /$ jts. 22541

Groleau, J. M., Calhoun, L. G., Cann, A., \& Tedeschi, R. G. (2013). The role of centrality of events in posttraumatic distress and posttraumatic growth. Psychological Trauma: Theory, Research, Practice, and Policy, 5(5), 477-483. https://doi.org/10.1037/a0028809

Kessler, R. C., Aguilar-Gaxiola, S., Alonso, J., Benjet, C., Bromet, E. J., Cardoso, G., \& Koenen, K. C. (2017). Trauma and PTSD in the WHO world mental health surveys. European Journal of Psychotraumatology, 8(sup5), 1353383-1353383. https://doi.org/ 10.1080/20008198.2017.1353383

Lancaster, S. L., Rodriguez, B. F., \& Weston, R. (2011). Path analytic examination of a cognitive model of PTSD. Behaviour Research and Therapy, 49(3), 194-201. https://doi.org/10.1016/j.brat.2011. 01.002

Liu, H., Petukhova, M. V., Sampson, N. A., Aguilar-Gaxiola, S., Alonso, J., Andrade, L. H., \& Kessler, R. C. (2017). Association of DSM-IV posttraumatic stress disorder with traumatic experience type and history in the World Health Organization World 
Mental Health Surveys. JAMA Psychiatry, 74(3), 270-281. https:// doi.org/10.1001/jamapsychiatry.2016.3783

McNally, R. J., \& Woud, M. L. (2019). Innovations in the study of appraisals and PTSD. A Commentary. Cognitive Therapy and Research, 43(1), 295-302. https://doi.org/10.1007/ s10608-018-09995-2

R Core Team. (2020). R: A language and environment for statistical computing. Vienna, Austria: R Foundation for Statistical Computing. Retrieved from https://www.R-project.org/.

Resick, P. A., Monson, C. M., \& Chard, K. M. (2017). Cognitive Processing Therapy for PTSD: A Comprehensive Manual. Guilford Press.

Rosseel, Y. (2012). Lavaan: An R package for structural equation modeling. Journal of Statistical Software, 48(2), 1-36. https://doi.org/ 10.18637/jss.v048.i02

Schad, D. J., Vasishth, S., Hohenstein, S., \& Kliegl, R. (2020). How to capitalize on a priori contrasts in linear (mixed) models: A tutorial. Journal of Memory and Language, 110, 104038. https://doi. org/10.1016/j.jml.2019.104038
Schuettler, D., \& Boals, A. (2011). The path to posttraumatic growth versus posttraumatic stress disorder: Contributions of event centrality and coping. Journal of Loss and Trauma, 16(2), 180-194. https://doi.org/10.1080/15325024.2010.519273

Teale Sapach, M. J. N., Horswill, S. C., Parkerson, H. A., Asmundson, G. J. G., \& Carleton, R. N. (2018). Centrality of traumatic events: Double edged sword or matter of valence? Cognitive Therapy and Research. https://doi.org/10.1007/s10608-018-9983-9

Tedeschi, R. G., \& Calhoun, L. G. (2004). Posttraumatic growth: Conceptual foundations and empirical evidence. Psychological Inquiry, 15(1), 1-18. https://doi.org/10.1207/s15327965pli1501_ 01

Vermeulen, M., Brown, A. D., Raes, F., \& Krans, J. (2019). Decreasing event centrality in undergraduates using cognitive bias modification of appraisals. Cognitive Therapy and Research, 43(1), 214-225. https://doi.org/10.1007/s10608-018-9936-3

World Health Organization. (1992). International statistical classification of diseases and related health problems (10th ed.). Author.

Publisher's Note Springer Nature remains neutral with regard to jurisdictional claims in published maps and institutional affiliations. 